\title{
Locomotion CONTROL Method FOR PATIENTS VERTICALIZATION WITH REGARD TO THEIR SAFETY AND COMFORT
}

\author{
Sergey Jatsun, Sergei Savin, Andrey Yatsun, Ruslan Turlapov \\ Southwest State University, 94, 50 let Oktyabrya, Kursk 305040, Russia
}

\begin{abstract}
This paper studies machine-assisted (mechanized) verticalization technology of patients. The control system is designed to assure the patient's safety by dealing with issues such as fall prevention and limits on joint angles' range of change. The patient's comfort is taken into account by imposing limits on the acceleration of different body parts of the patient. The control system should be tuned with respect to each patient's specific requirements, because the mentioned limits are different for different people and may depend on the type of the patient's illness. To realize this, we study the admissible region of control system parameters. The control system is designed such that the center of mass of the human-machine system moves along a certain trajectory with the possibility of controlling its final position and velocity. The influence of various parameters on the mechanism's trajectory and balance is discussed. Regions of parameters that allow the system to stand up without tipping over are presented.
\end{abstract}

Keywords: exoskeleton; locomotion control; areas of parameters
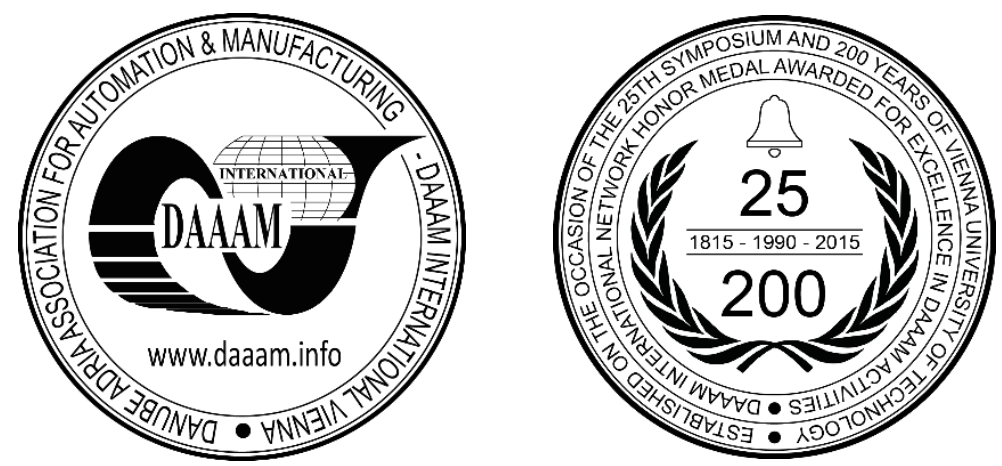

This Publication has to be referred as: Jatsun, S[ergey]; Savin, S[ergei]; Yatsun, A[ndrey] \& Turlapov, R[uslan] (2016). Locomotion Control Method for Patients Verticalization with Regard to their Safety and Comfort, Proceedings of the 26th DAAAM International Symposium, pp.1129-1137, B. Katalinic (Ed.), Published by DAAAM International, ISBN 978-3-902734-07-5, ISSN 1726-9679, Vienna, Austria DOI: $10.2507 / 26$ th.daaam.proceedings.159 


\section{Introduction}

Medical treatment and rehabilitation procedures for patients with locomotive disorders often involve the need to raise the patient to a standing position. People nowadays rely mainly on personal assistants to perform this task. It is clear that automation of this procedure is needed based on considerations of cost, safety and availability of assistants. Active research work being done in this field also indicates the importance of this issue [1-3, 14-16, 20].

There are two main approaches to the automation of patient verticalization. The first approach relies on the use of an external device (assistant robot or special equipment) which takes up the role of a human assistant [1-3, 20]. The second approach exploits exoskeleton technology that is currently demonstrating significant progress [14-16]. A new generation of exoskeletons that allows a person to move even in the case of damage of lower extremities recently came into service. Also, medical research indicates that performing motion with an exoskeleton can speed up recovery of people with certain types of temporal locomotive disorders.

The development and production of verticalization exoskeletons requires well-developed theory of the functioning of such systems with particular emphasis on dynamics and control. The common problems in the theory of walking mechanisms have been developed in [4-13] and other publications. The focus of these publications is controlled walking, which includes a wide range of problems such as gait generation and motion planning [4-6, 8, 13], stability [5, 7] and obstacle negotiation [11, 12]. Papers [24-26] propose methods to enhance the performance of the walkng systems. At the same time we should note that for a normal person walking is preceded by the process of sit-to-stand. The problem of performing controllable sit-to-stand motion with regards to the patient's safety and comfort is not well studied. There are a number of medical and technical papers that discuss the different aspects of sit-to-stand motion [14-20]. In papers [14-16] the use of robot suits that help paraplegic patients [14-15] and elderly people [16] to stand is discussed. Papers $[17,18]$ analyse the use of fuzzy logic applications in exoskeletons performing this kind of motion with emphasis on the use of wearable sensors. Paper [20] discusses the use of an assistant robot that helps a person to stand up. In paper [21] experimental data obtained from studying sit-to-stand motion of a person is shown and different methods of approximating it by smooth functions are compared.

At the same time the important issue of limiting the accelerations experienced by the patient and the joint angles' ranges of change, while simultaneously preserving stability of the mechanism have not been sufficiently studied. Limiting accelerations is connected with the patient's comfort, while limiting the ranges of change of joint angles and maintaining stability of the mechanism are connected with the patient's safety. So the main purpose of the study is investigation of the person motion wearing an exoskeleton during sit-to-stand motion

\section{Research object}

In this work, we focus on the mechanism's controlled motion during the process of rising i.e. vertical displacement of the center of mass. Let us look at the mechanism's analytical diagram shown in Fig.1. The mechanism consists of four links in the form of uniform rigid rods of length $l_{i}$ and mass $m_{i}(i=1 . .4)$.

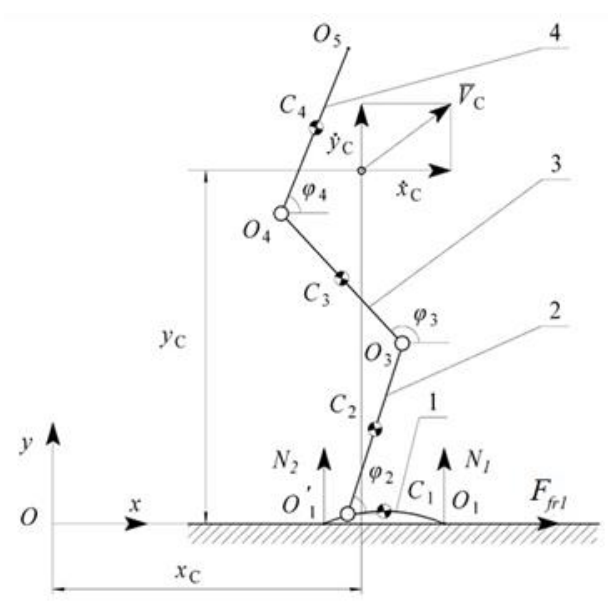

Fig. 1. Simplified dynamic scheme of the robotic exoskeleton. 1- 4 - first- fourth links of the mechanism

The centers of mass are located at points $C_{i}$. The links are joined to each other at points $O_{2}, O_{3}$ and $O_{4}$, where the electric drives that generate torques: $M_{1}, M_{2}$ and $M_{3}$ are installed. Link 1 is on a rough surface, with contact taking place at points $O_{1}$ and $O_{2}$. Forces: $\vec{N}_{1}, \vec{N}_{2}$ and $\vec{F}_{f r 1}$, applied at points $O_{1}$ and $O_{2}$ arise due to this interaction as shown in the diagram. Forces of gravity, $m_{i} \vec{g}$ also act on the system. 
Fig. 1 shows a stationary coordinate system, $O_{x y}$ (further system (0)). We introduce a vector of generalized coordinates: $\vec{q}=\left[\begin{array}{lll}\varphi_{2} & \varphi_{3} & \varphi_{4}\end{array}\right]^{\mathrm{T}}$, where $\varphi_{i}$ is the angle between links $O_{i} O_{i+1}$ and the Ox axis. Thus the unknowns are the functions: $\varphi_{2}(t), \varphi_{3}(t), \varphi_{4}(t), N_{1}(t), N_{2}(t)$ and $F_{f r 1}(t)$.

Let us consider the case when the foot remains stationary i.e. none of the contact points separate from the surface and point $\mathrm{O} 1$ does not slide. For the given case, $N_{1}, N_{2}$ and $F_{f r 1}$ are limited as follows:

$$
N_{1}>0, N_{2}>0,\left|F_{f r 1}\right| \leq F_{f r 1}^{0}
$$

where $F_{m p 1}^{0}$ is the maximum value of static friction.

\section{Mathematical model of the mechanism}

In order to write the mechanism's equations of dynamics we use the Lagrange equation with constraints. These equations can be written in general form as follows:

$$
\frac{d}{d t} \frac{\partial \mathrm{T}}{\partial \dot{q}_{i}}-\frac{\partial \mathrm{T}}{\partial q_{i}}=-\frac{\partial \Pi}{\partial q_{i}}+Q_{i}+\sum_{j=1}^{m} \lambda_{j} \frac{\partial h_{j}}{\partial q_{i}}, i=\overline{1,6}
$$

where $\mathrm{T}$ is the total kinetic energy, $\Pi$ - the potential energy, $Q_{i}-$ the $\mathrm{i}$-th generalized force, $h_{j}-$ the equation of the $\mathrm{j}$-th constraint, $\lambda_{j}$ - the Lagrange multiplier corresponding to the $\mathrm{j}$-th constraint.

The equations of constraints imposed on the four-link mechanism by the surface are as follows:

$$
h=\left[\begin{array}{c}
x_{O 1} \\
y_{O 1} \\
y_{O 1}+l_{1} \sin \left(\varphi_{1}\right)
\end{array}\right]=\left[\begin{array}{l}
0 \\
0 \\
0
\end{array}\right]
$$

where $x_{O 1}, y_{O 1}$ are the coordinates of point $O_{1}$ of the first links.

Taking partial derivatives in (2), we obtain the mechanism's equations of motion. After differentiating and writing the equations in the vector form used in works [6-8] we get:

$$
\mathrm{A}(\varphi) \ddot{\varphi}+\vec{b}(\varphi, \dot{\varphi})+\vec{G}(\varphi)+\vec{f}(\dot{\varphi})=J(\varphi) \bar{\lambda}+\vec{\Gamma}
$$

where $\mathrm{A}(\varphi)$ is the joint space mass inertia matrix, $\vec{b}(\varphi, \dot{\varphi})$ - the vector of centrifugal and Coriolis forces, $\vec{G}(\varphi)-$ the vector of potential forces, $\vec{f}(\dot{\varphi})$ - the vector of dissipative forces obtained by differentiating Rayleigh's dissipative function, $J(\varphi)$ - the matrix of partial derivatives standing before the Lagrange multipliers, $\vec{\lambda}$ - the vector of Lagrange multipliers, $\vec{\Gamma}$ - the vector of generalized forces.

After introducing dimensionless coefficients we rewrite the equations in the following form (following equations are written down explicitly in [23]):

$$
\begin{aligned}
& \ddot{\varphi}_{2}+a_{11} \ddot{\varphi}_{3} \cos \left(\varphi_{2}-\varphi_{3}\right)+a_{12} \ddot{\varphi}_{4} \cos \left(\varphi_{2}-\varphi_{4}\right)+a_{11} \dot{\varphi}_{3}^{2} \sin \left(\varphi_{2}-\varphi_{3}\right)+a_{12} \dot{\varphi}_{4}^{2} \sin \left(\varphi_{2}-\varphi_{4}\right)= \\
& =-a_{14} M_{21}-a_{13} \cos \varphi_{2}-a_{14}\left(\mu_{2}\left(\dot{\varphi}_{2}-\dot{\varphi}_{3}\right)+\mu_{3} \dot{\varphi}_{2}+k_{2}\left(\varphi_{2}-\varphi_{3}\right)+k_{3} \varphi_{2}\right) \\
& \ddot{\varphi}_{3}+a_{21} \ddot{\varphi}_{2} \cos \left(\varphi_{3}-\varphi_{2}\right)+a_{22} \ddot{\varphi}_{4} \cos \left(\varphi_{3}-\varphi_{4}\right)+a_{21} \dot{\varphi}_{2}^{2} \sin \left(\varphi_{3}-\varphi_{2}\right)+a_{22} \dot{\varphi}_{4}^{2} \sin \left(\varphi_{3}-\varphi_{4}\right)= \\
& a_{24}\left(M_{12}-M_{32}\right)-a_{23} \cos \varphi_{3}-a_{24}\left(\mu_{1}\left(\dot{\varphi}_{3}-\dot{\varphi}_{4}\right)+\mu_{2}\left(\dot{\varphi}_{3}-\dot{\varphi}_{2}\right)+k_{1}\left(\varphi_{3}-\varphi_{4}\right)+k_{2}\left(\varphi_{3}-\varphi_{2}\right)\right) \\
& \ddot{\varphi}_{4}+a_{31} \ddot{\varphi}_{2} \cos \left(\varphi_{4}-\varphi_{2}\right)+a_{32} \ddot{\varphi}_{3} \cos \left(\varphi_{4}-\varphi_{3}\right)+a_{31} \dot{\varphi}_{2}^{2} \sin \left(\varphi_{4}-\varphi_{3}\right)+a_{32} \dot{\varphi}_{2}^{2} \sin \left(\varphi_{4}-\varphi_{3}\right)= \\
& =a_{34}\left(M_{23}-M_{43}\right)-a_{33} \cos \varphi_{4}-a_{34}\left(\mu_{1}\left(\dot{\varphi}_{4}-\dot{\varphi}_{3}\right)+k_{1}\left(\varphi_{4}-\varphi_{3}\right)\right)
\end{aligned}
$$


where:

$$
\begin{aligned}
& a_{11}=\frac{\left(m_{3}+2 m_{4}\right) l_{2} l_{3}}{2 J_{2}+2\left(m_{3}+m_{4}\right) l_{2}^{2}} \quad a_{12}=\frac{m_{4} l_{2} l_{4}}{2 J_{2}+2\left(m_{3}+m_{4}\right) l_{2}^{2}}, \quad a_{13}=\frac{\left(m_{2}+2 m_{3}+2 m_{4}\right) g l_{2}}{2 J_{2}+2\left(m_{3}+m_{4}\right) l_{2}^{2}} \quad a_{14}=\frac{1}{J_{2}+\left(m_{3}+m_{4}\right) l_{2}^{2}}, \\
& a_{21}=\frac{\left(m_{3}+2 m_{4}\right) l_{2} l_{3}}{2 J_{3}+0.5 m_{3} l_{3}^{2}+2 m_{4} l_{3}^{2}}, a_{22}=\frac{2 m_{4} l_{3} l_{4}}{4 J_{3}+m_{3} l_{3}^{2}+4 m_{4} l_{3}^{2}}, \quad a_{23}=\frac{\left(m_{3}+2 m_{4}\right) g l_{3}}{2 J_{3}+0.5 m_{3} l_{3}^{2}+2 m_{4} l_{3}^{2}}, \quad a_{24}=\frac{4}{4 J_{3}+m_{3} l_{3}^{2}+4 m_{4} l_{3}^{2}}, \\
& a_{31}=\frac{2 m_{4} l_{2} l_{4}}{4 J_{4}+m_{4} l_{4}^{2}}, a_{32}=\frac{2 m_{4} l_{3} l_{4}}{4 J_{4}+m_{4} l_{4}^{2}}, a_{33}=\frac{2 m_{4} g l_{4}}{4 J_{4}+m_{4} l_{4}^{2}}, \quad a_{34}=\frac{4}{4 J_{4}+m_{4} l_{4}^{2}} .
\end{aligned}
$$

From equations (5) we obtain the expressions for constraints: $N_{1}, N_{2}$ and $F_{f r 1}$ which coincide in magnitude with the components of vector $\overleftarrow{\lambda}$

$$
\begin{aligned}
& N_{1}=\left(\sum_{i=2}^{4} m_{i}-\frac{m_{2}}{2}\right) \cdot l_{2} \cdot\left(\ddot{\varphi}_{2}^{2} \cdot \sin \left(\varphi_{1}\right)+\dot{\varphi}_{1}^{2} \cdot \cos \left(\varphi_{2}\right)\right)+l_{3}\left(\frac{m_{3}}{2}+m_{4}\right)\left(\ddot{\varphi}_{3}^{2} \cdot \sin \left(\varphi_{2}\right)+\dot{\varphi}_{2}^{2} \cdot \cos \left(\varphi_{2}\right)\right)+ \\
& +\frac{m_{4} \cdot l_{4}}{2}\left(\ddot{\varphi}_{4}^{2} \cdot \sin \left(\varphi_{3}\right)+\dot{\varphi}_{3}^{2} \cdot \cos \left(\varphi_{3}\right)\right) \\
& N_{2}=\left(\sum_{i=2}^{4} m_{i}-\frac{m_{2}}{2}\right) \cdot l_{2} \cdot\left(\ddot{\varphi}_{2}^{2} \cdot \cos \left(\varphi_{1}\right)-\dot{\varphi}_{1}^{2} \cdot \sin \left(\varphi_{1}\right)\right)+\left(\frac{m_{3}}{2}+m_{4}\right) l_{3} \cdot\left(\ddot{\varphi}_{3}^{2} \cdot \cos \left(\varphi_{2}\right)-\dot{\varphi}_{2}{ }^{2} \cdot \sin \left(\varphi_{2}\right)\right) \\
& \frac{m_{4} \cdot l_{4}}{2}\left(\ddot{\varphi}_{4}^{2} \cdot \cos \left(\varphi_{3}\right)-\dot{\varphi}_{3}{ }^{2} \cdot \sin \left(\varphi_{3}\right)\right)+\sum_{i=1}^{4} m_{i} g \\
& F_{m p l}=\left(\sum_{i=2}^{4} m_{i}-\frac{m_{2}}{2}\right) \frac{l_{2}}{l_{1}}\left(\left(1+l_{1} \cos \left(\varphi_{1}\right)\right) \cdot \cos \left(\varphi_{1}\right) \ddot{\varphi}_{2}^{2}+\left(l_{1}-1\right) \dot{\varphi}_{1}^{2} \sin \left(\varphi_{1}\right)\right)+ \\
& +\frac{m_{3}+2 m_{4}}{2 l_{1}} \cdot l_{3} \cdot\left[\left(l_{1}+1\right) \cdot \cos \left(\varphi_{2}\right) \cdot \ddot{\varphi}_{3}^{2}+\left(l_{1}-1\right) \cdot \dot{\varphi}_{2}{ }^{2} \sin \left(\varphi_{2}\right)\right]+ \\
& +\frac{m_{4} \cdot l_{4}}{2 l_{1}} \cdot\left(\left(l_{1}+1\right) \cdot \cos \left(\varphi_{3}\right) \cdot \ddot{\varphi}_{4}^{2}+\left(l_{1}-1\right) \cdot \dot{\varphi}_{3}^{2} \sin \left(\varphi_{3}\right)\right)+\left(\sum_{i=1}^{4} m_{i}\left(\frac{1}{l_{1}}+1\right)-\frac{m_{1}}{2}\right) g-\frac{M_{1}-\mu_{1} \dot{\varphi}_{1}}{l_{1}}
\end{aligned}
$$

The obtained system of algebraic and differential equations allows us to determine the functions, ${ }_{i}(t)$, as well as the time functions of the values of reactions from the supporting surface. We can also determine the values of the torques required for realizing a given trajectory of the device's links.

\section{Automatic control system}

In this work we do not consider the dynamics of the electric drive supposing that the regulator can directly control its torque. We shall be using the exoskeleton's control system structure proposed in publication [22]. Work [22] presents a method that allows us to obtain the desired values of generalized coordinates as time functions in such a way that they would provide motion of the mechanism's center of mass along a given trajectory. The position of the exoskeleton's center of mass can be written in the form:

$$
\left[\begin{array}{l}
x_{C} \\
y_{C}
\end{array}\right]=\left[\begin{array}{c}
x_{O 1}+\sum_{i=1}^{4} K_{i} \cos \left(\varphi_{i}\right) \\
y_{O 1}+\sum_{i=1}^{4} K_{i} \sin \left(\varphi_{i}\right)
\end{array}\right]
$$

where:

$$
K_{1}=l_{1} \frac{\sum_{i=1}^{4} m_{i}-0.5 m_{1}}{\sum_{i=1}^{4} m_{i}} \quad K_{2}=l_{2} \frac{\sum_{i=2}^{4} m_{i}-0.5 m_{2}}{\sum_{i=1}^{4} m_{i}} \quad K_{3}=l_{3} \frac{0.5 m_{3}+m_{4}}{\sum_{i=1}^{4} m_{i}} \quad K_{4}=\frac{0.5 l_{4} m_{4}}{\sum_{i=1}^{4} m_{i}} .
$$


Let the desired trajectory of the center of mass be described by the equations:

$$
x_{C}(t)=a_{0}, \quad y_{C}(t)=a_{1} \cdot t^{3}+a_{2} \cdot t^{2}+a_{3} \cdot t+a_{4},
$$

Then the time functions of generalized coordinates $-\varphi_{1}$ and $\varphi_{2}$ that enable the realization of this trajectory considering constraints (6) are as follows:

$$
\begin{aligned}
& \varphi_{2}=-\arccos \left(\frac{b_{1}^{2}+b_{2}^{2}+K_{1}^{2}-K_{2}^{2}}{2 K_{1} \sqrt{b_{1}^{2}+b_{2}^{2}}}\right)+\arccos \left(\frac{b_{1}}{\sqrt{b_{1}^{2}+b_{2}^{2}}}\right) \\
& \varphi_{3}=\arccos \left(\frac{b_{1}^{2}+b_{2}^{2}+K_{2}^{2}-K_{1}^{2}}{2 K_{2} \sqrt{b_{1}^{2}+b_{2}^{2}}}\right)+\arccos \left(\frac{b_{1}}{\sqrt{b_{1}^{2}+b_{2}^{2}}}\right),
\end{aligned}
$$

$$
\text { where } b_{1}=a_{0}-K_{1}-K_{4} \cos \left(\varphi_{4}\right), b_{2}=a_{1} \cdot t^{3}+a_{2} \cdot t^{2}+a_{3} \cdot t+a_{4}-K_{4} \sin \left(\varphi_{4}\right)
$$

The time function of $\varphi_{4}$ is given arbitrarily. In order to evaluate the constants, $a_{i}$ the initial and final conditions and the speed of the center of mass are given as projections on the coordinate axes. Here we consider a control strategy when initially the center of mass is stationary, and the desired value of its velocity along horizontal axis equals zero during the whole process of verticalization.

We denote the time in which the mechanism moves from the initial to the final position as $t_{f}$. We note that at time $t_{f}$ the value of the center of mass' velocity along vertical axis can be either zero or nonzero (see Fig. 2). The latter case corresponds to the acceleration mode before a jump.

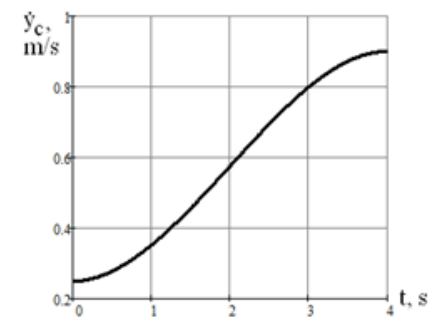

a)

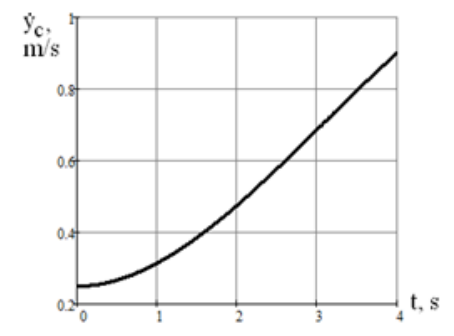

b)

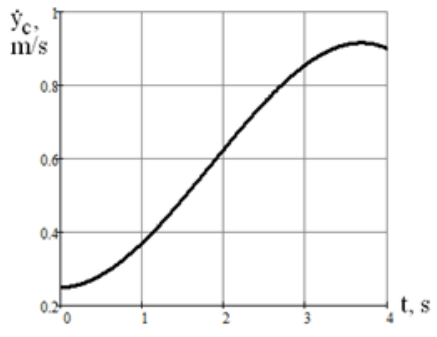

c)

Fig. 2. Different versions of the time functions of the center of mass' velocity a) velocity at time $t_{f}$ equals zero, b) and c) the center of mass' velocity at time $t_{f}$ is nonzero

Let us look at the problem of organizing vertical displacement of the device's center of mass such that the robot's foot does not lose contact with the supporting surface. The nullification of normal reactions- $\mathrm{N}_{1}$ and $\mathrm{N}_{2}$ shall be considered as loss of contact. The flow diagram of the modelled system is shown in Fig. 3..

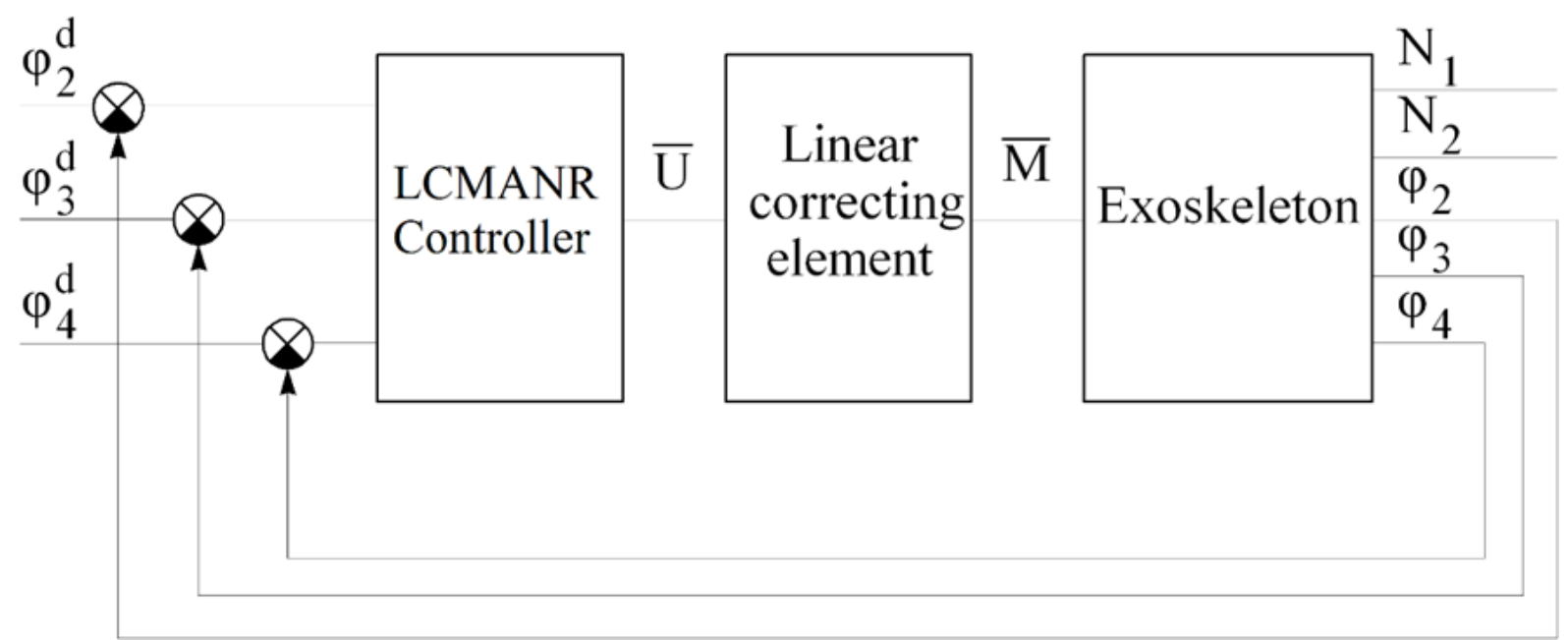

Fig. 3. Exoskeleton's control system scheme 
In the Fig. 3 LCMANR means Locomotion Control Method limiting Accelerations and Normal Reactions.

Let us consider the task of determining the region of parameters of the given trajectory of motion of the center of mass (12) in which verticalization is possible without nullification of normal reactions. We note that the parameters of polynomials (12) are determined from the boundary conditions (the center of mass' initial and final positions):

$$
\begin{aligned}
& a_{1}=\frac{2}{t_{f}^{3}}\left(y_{C}(0)-y_{C}\left(t_{f}\right)\right)+\frac{2}{t_{f}^{2}}\left(\dot{y}_{C}(0)+\dot{y}_{C}\left(t_{f}\right)\right) \\
& a_{2}=\frac{3}{t_{f}^{2}}\left(y_{C}\left(t_{f}\right)-y_{C}(0)\right)-\frac{1}{t_{f}}\left(2 \dot{y}_{C}(0)+\dot{y}_{C}\left(t_{f}\right)\right) \\
& a_{3}=\dot{y}_{C}(0) \quad a_{4}=y_{C}(0)
\end{aligned}
$$

where $t_{f}$ is the time in which verticalization is implemented.

We introduce the denotations: $y_{C}\left(t_{f}\right)=Y_{1}$ and $\dot{y}_{C}\left(t_{f}\right)=\dot{Y}_{1}$. We find the area of parameters $Y_{1}$ and $\dot{Y}_{1}$, in which verticalization is possible without the nullification of reactions $N_{1}$ and $N_{2}$ (see Fig. 4).

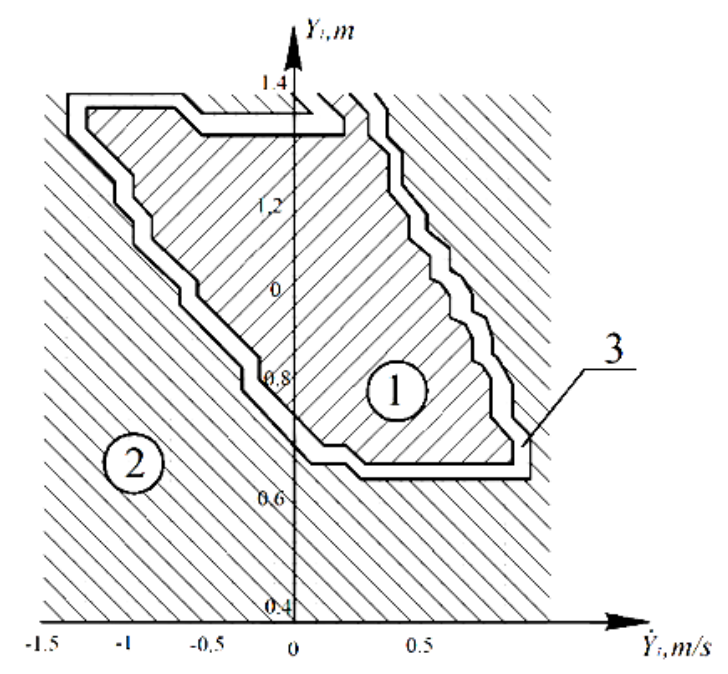

Fig. 4. Area of parameters $Y_{1}$ and $\dot{Y}_{1} ; 1-$ area where $N_{1}(t)>0$ and $N_{2}(t)>0$ in the whole time interval: $t \in\left\lfloor\begin{array}{ll}0 & t_{f}\end{array}\right\rfloor, 2$

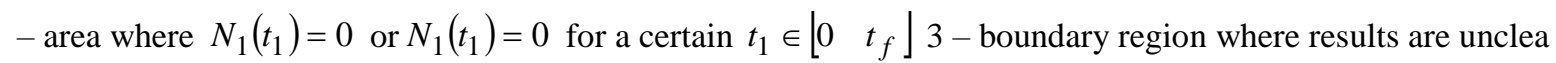

The upper and lower boundaries of the obtained region are explained by human physiological capabilities and the mechanism's structural peculiarities that impose limitations on the minimum and maximum possible heights of the system's center of mass. The right and left boundaries of the region of parameters allow us to determine the maximum permissible speed of the center of mass at the end of motion. The practical meaning of the right boundary consists in that it separates "rising" and "jumping". The region to the left of the ordinate axis shows the motion during which the velocity of the center of mass is governed by the law shown in fig. 2 (c). During such motion regime the center of mass descends at time $t_{f}$. The left boundary of region 1 shows the velocity of descent which can be given so that the mechanism does not lose contact with the surface.

The obtained results allow us to make a conclusion on the possibility of defining the trajectory of motion of the center of mass by lower degree polynomials without controlling its velocity at the end of virtualization. It is sufficient to know whether or not parameters $Y_{1}$ and $\dot{Y}_{1}$, characterizing the selected trajectory belong to the region 1 .

Since the values of $a_{1}$ and $a_{2}$ depend linearly on $Y_{1}$ and $\dot{Y}_{1}$, we can reconstruct the area shown in fig.5 in the space of parameters $a_{1}$ and $a_{2}$. The result is shown in fig. 6 


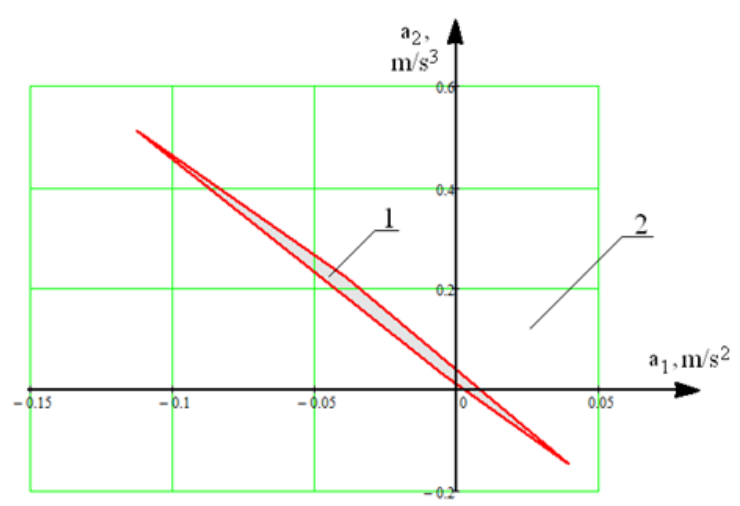

Fig. 5. Area of parameters $a_{1}$ and $a_{2} ; 1-$ area where $N_{1}(t)>0$ and $N_{2}(t)>0$ in the whole time interval: $t \in\left[\begin{array}{ll}0 & t_{f}\end{array}\right], 2$

$$
\text { - area where } N_{1}\left(t_{1}\right)=0 \text { or } N_{1}\left(t_{1}\right)=0 \text { for a certain } t_{1} \in\left[\begin{array}{ll}
0 & t_{f}
\end{array}\right]
$$

The shown area of parameters is similar to a rhombus stretched along a line. The obtained region can be used in cases when the assignment of the coefficients of the polynomials describing the required trajectory of the center of mass is done directly. We can conclude that a relatively small change in the values of each of the coefficients, $a_{1}$ and $a_{2}$ can take the system out of region 1 i.e. lead to detachment of the foot from the surface during rising. This can be avoided by choosing coefficients near the line described above.

We can now consider the regions of parameters where acceleration of the patient's head and center of mass of do not exceed certain limits. To do this we need to plot acceleration distributions.

Since $y_{C}(t)$ is a third order polynomial its second derivative is a linear function in $t$. This means that it has no local extrema and its maximum absolute value can only lie on the boundary of its domain. Assuming that the desired and real values for $\max \left(\ddot{y}_{C}(t)\right)$ are the same we can use the following expression:

$$
\left.\max _{t \in[0} \ddot{y}_{t_{1}}\right] \ddot{y}_{C}(t)=\max \left\{2 a_{2}, \quad 6 a_{1} t_{1}+2 a_{2}\right\}
$$

The acceleration distributions of the center of mass are shown in fig. 6.

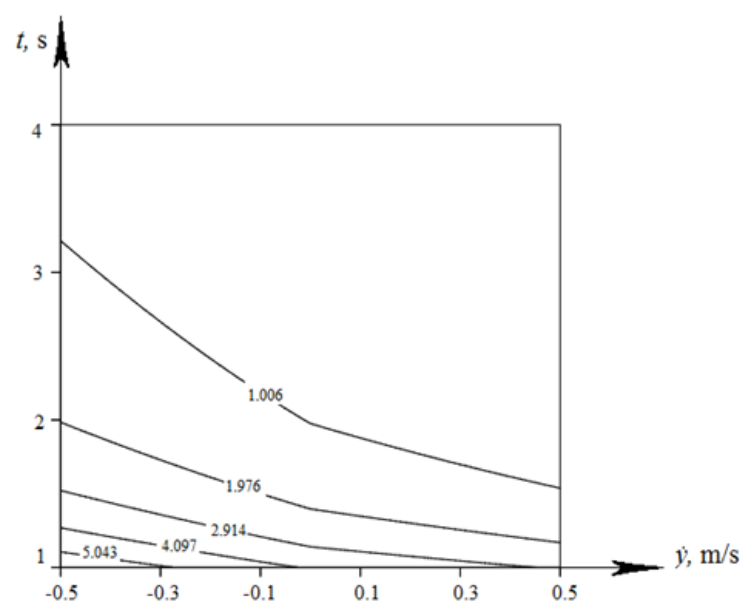

Fig. 6. Acceleration distributions for the center of mass

The counter lines of the Fig. 6 separate parameter regions based on maximum accelerations the center of mass should have for their parameter values.

To find absolute value of acceleration of the head we need use the next formula:

$$
a_{h}=\left(\left[\sum_{i=2}^{4} L_{i}\left(\ddot{\varphi}_{i} \sin \left(\varphi_{i}\right)+\dot{\varphi}_{i}^{2} \cos \left(\varphi_{i}\right)\right)\right]^{2}+\left[\sum_{i=2}^{4} L_{i}\left(\ddot{\varphi}_{i} \cos \left(\varphi_{i}\right)-\dot{\varphi}_{i}^{2} \sin \left(\varphi_{i}\right)\right)\right]^{2}\right)^{1 / 2}
$$


where $L_{4}$ is the distance between the hip joint and the head and $L_{2}=l_{2}, L_{3}=l_{3}$. In this work we find numerically the maximum value of this function in the time interval: $t \in\left[\begin{array}{ll}0 & t_{1}\end{array}\right]$. The resulting regions of parameters are shown in Fig. 7

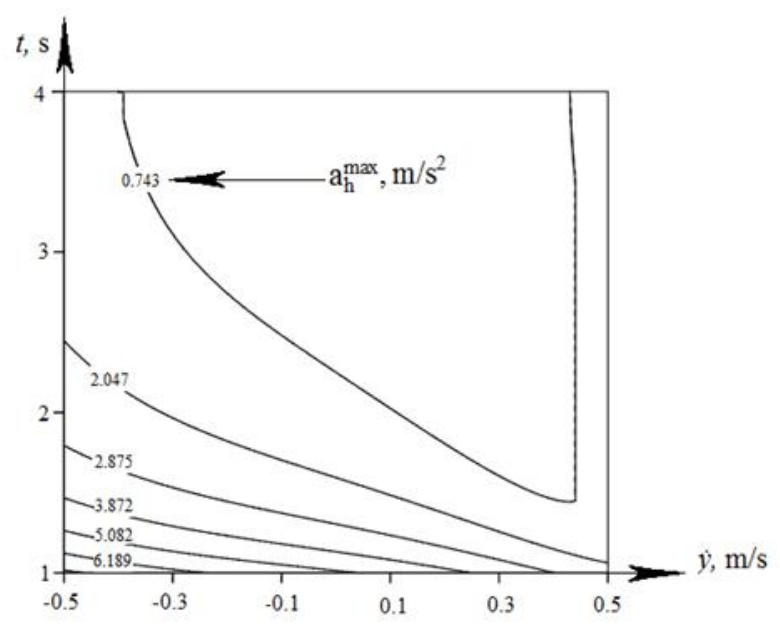

Fig. 7. Acceleration distributions for the head

Although the shapes of counter lines on Figs. 6 and 7 are different, we notice a general similarity. The obtained graphs present a way of taking into account the patient's comfort during the stage of designing control tasks.

\section{Conclusion}

In this paper, the exoskeleton system used for patient's verticalization was considered. We use approach for normal reaction force's values control to achieve balance of the system. Accelerations of patient's head and center of mass were controlled. For the case when the exoskeleton is performing standing up motion by moving its center of mass along a given trajectory, admissible regions for control system's parameters were obtained. Different ways of representing this area were shown, the advantage of using one of them was discussed. Influence that parameters have on normal reactions and accelerations of the center of mass and the head give information about safety and comfort for the user.

\section{Acknowledgements}

Work is performed with RSF, Project № 14-39-00008 "The establishment of the research laboratory of modern methods and robotic systems to improve the human environment".

\section{References}

[1] Chugo, Daisuke, Kuniaki Kawabata, Hiroyuki Okamoto, Hayato Kaetsu, Hajime Asama, Norihisa Miyake, and Kazuhiro Kosuge. "Force assistance system for standing-up motion." Industrial Robot: An International Journal 34, no. 2 (2007): 128-134.

[2] Kuznetsov, Alexey N., Natalia V. Rybalko, Vadim D. Daminov, and Andreas R. Luft. "Early poststroke rehabilitation using a robotic tilt-table stepper and functional electrical stimulation." Stroke research and treatment 2013 (2013).

[3] Calabrò, Rocco Salvatore, Antonino Naro, Margherita Russo, Antonino Leo, Tina Balletta, Ileana Saccà, Rosaria De Luca, and Placido Bramanti. "Do post-stroke patients benefit from robotic verticalization? A pilot-study focusing on a novel neurophysiological approach." Restorative Neurology and Neuroscience Preprint: 1-11, 8 August 2015.

[4] Kajita, S., Kanehiro, F., Kaneko, K., Yokoi, K., \& Hirukawa, H. (2001). The 3D Linear Inverted Pendulum Mode: A simple modeling for a biped walking pattern generation. In Intelligent Robots and Systems, 2001. Proceedings. 2001 IEEE/RSJ International Conference on (Vol. 1, pp. 239-246). IEEE.

[5] Kajita, S., Kanehiro, F., Kaneko, K., Fujiwara, K., Harada, K., Yokoi, K., \& Hirukawa, H. (2003, September). Biped walking pattern generation by using preview control of zero-moment point. In Robotics and Automation, 2003. Proceedings. ICRA'03. IEEE International Conference on (Vol. 2, pp. 1620-1626). IEEE.

[6] Harada, K., Kajita, S., Kanehiro, F., Fujiwara, K., Kaneko, K., Yokoi, K., \& Hirukawa, H. (2007). Real-time planning of humanoid robot's gait for force-controlled manipulation. Mechatronics, IEEE/ASME Transactions on, 12(1), 53-62.

[7] Kajita, S., Morisawa, M., Miura, K., Nakaoka, S., Harada, K., Kaneko, K., ... \& Yokoi, K. (2010, October). Biped walking stabilization based on linear inverted pendulum tracking. In Intelligent Robots and Systems (IROS), 2010 IEEE/RSJ International Conference on (pp. 4489-4496). IEEE. 
[8] Huang, Q., Yokoi, K., Kajita, S., Kaneko, K., Arai, H., Koyachi, N., \& Tanie, K. (2001). Planning walking patterns for a biped robot. Robotics and Automation, IEEE Transactions on, 17(3), 280-289.

[9] Sellaouti, R., Stasse, O., Kajita, S., Yokoi, K., \& Kheddar, A. (2006, October). Faster and smoother walking of humanoid HRP-2 with passive toe joints. In Intelligent Robots and Systems, 2006 IEEE/RSJ International Conference on (pp. 4909-4914). IEEE.

[10] Arisumi, H., Miossec, S., Chardonnet, J. R., \& Yokoi, K. (2008, September). Dynamic lifting by whole body motion of humanoid robots. In Intelligent Robots and Systems, 2008. IROS 2008. IEEE/RSJ International Conference on (pp. 668-675). IEEE.

[11] Yoshida, E., Esteves, C., Sakaguchi, T., Laumond, J. P., \& Yokoi, K. (2006, October). Smooth collision avoidance: Practical issues in dynamic humanoid motion. In Intelligent Robots and Systems, 2006 IEEE/RSJ International Conference on (pp. 827-832). IEEE.

[12] Verrelst, B., Stasse, O., Yokoi, K., \& Vanderborght, B. (2006, December). Dynamically stepping over obstacles by the humanoid robot HRP-2. In Humanoid Robots, 2006 6th IEEE-RAS International Conference on (pp. 117-123). IEEE.

[13] Brock, O., \& Khatib, O. (2002). Elastic strips: A framework for motion generation in human environments. The International Journal of Robotics Research, 21(12), 1031-1052.

[14] Tsukahara, A., Hasegawa, Y., \& Sankai, Y. (2009, June). Standing-up motion support for paraplegic patient with Robot Suit HAL. In Rehabilitation Robotics, 2009. ICORR 2009. IEEE International Conference on (pp. 211-217). IEEE.

[15] Tsukahara, A., Kawanishi, R., Hasegawa, Y., \& Sankai, Y. (2010). Sit-to-stand and stand-to-sit transfer support for complete paraplegic patients with robot suit HAL. Advanced robotics, 24(11), 1615-1638.

[16] Jun, H. G., Chang, Y. Y., Dan, B. J., Jo, B. R., Min, B. H., Yang, H., ... \& Kim, J. (2011, June). Walking and sit-tostand support system for elderly and disabled. In Rehabilitation Robotics (ICORR), 2011 IEEE International Conference on (pp. 1-5). IEEE.

[17] Taslim Reza, S. M., Ahmad, N., Choudhury, I. A., \& Ghazilla, R. A. R. (2014). A Fuzzy Controller for Lower Limb Exoskeletons during Sit-to-Stand and Stand-to-Sit Movement Using Wearable Sensors. Sensors, 14(3), $4342-4363$.

[18] Salah, O., Ramadan, A. A., Sessa, S., Ismail, A. A., Fujie, M., \& Takanishi, A. (2013). Anfis-based sensor fusion system of sit-to-stand for elderly people assistive device protocols. International Journal of Automation and Computing, 10(5), 405-413.

[19] Mughal, A. M., \& Iqbal, K. (2010, October). 3D bipedal model for biomechanical sit-to-stand movement with coupled torque optimization and experimental analysis. In Systems Man and Cybernetics (SMC), 2010 IEEE International Conference on (pp. 568-573). IEEE.

[20] López, A. M., Vaillant, J., Keith, F., Fraisse, P., \& Kheddar, A. (2014, November). Compliant control of a humanoid robot helping a person stand up from a seated position. In Humanoid Robots (Humanoids), 2014 14th IEEE-RAS International Conference on (pp. 817-822). IEEE.

[21] Jatsun S., Savin S., Bezmen P. Modelling of exoskeleton movement in verticalization process, New Developments in Pure and Applied Mathematics p.83-87, 2015.

[22] S. Jatsun, S. Savin, A. Yatsun, A. Malchikov Study of controlled motion of exoskeleton moving from sitting to standing position / "24th International Conference on Robotics in Alpe-Adria-Danube Region", 201.

[23] Jatsun S.F. The modelling of the standing-up process of the anthropomorphic mechanism / Jatsun S.F., Vorochaeva L. Yu., Yatsun A.S., Savin S.I. Proceedings of the International Conference on CLAWAR 2015, pp. 175-182.

[24] Englsberger, Johannes, Christian Ott, and Alin Albu-Schaffer. "Three-Dimensional Bipedal Walking Control Based on Divergent Component of Motion." Robotics, IEEE Transactions on 31, no. 2 (2015): 355-368.

[25] Shin, Hyeok-Ki, and Byung Kook Kim. "Energy-Efficient Gait Planning and Control for Biped Robots Utilizing the Allowable ZMP Region." Robotics, IEEE Transactions on 30, no. 4 (2014): 986-993.

[26] Geng, Tao. "Torso Inclination Enables Faster Walking in a Planar Biped Robot With Passive Ankles." Robotics, IEEE Transactions on 30, no. 3 (2014): 753-758. 